\title{
PERCEPÇÕES DO CONCEITO DE QUALIDADE NO DESIGN DE PRODUTOS PLÁSTICOS NAS VISÕES DE USUÁRIOS E DESIGNERS
}

Maria do Rosário Gonçalves Mira

Faculdade de Arquitetura e Urbanismo da Universidade de São Paulo

mariarosariomira@usp.br

Luís Cláudio Portugal do Nascimento

Faculdade de Arquitetura e Urbanismo da Universidade de São Paulo

claudioportugal@usp.br

Resumo: Este artigo apresenta resultados de pesquisa de mestrado concluída em 2015, realizada com usuários e designers profissionais, em pesquisa de natureza fenomenológica, a respeito da percepção das qualidades do material plástico aplicados ao design de produtos. Neste estudo, fez-se uso da técnica de entrevistas em profundidade semiestruturadas, realizadas com 35 participantes (usuários) e doze designers, homens e mulheres, em idades variadas, em amostra não estratificada. Para o tratamento de dados, utilizou-se da técnica de análise associativa de dados, baseada no processo de identificação indutiva de eixos temáticos, a partir do exame analítico dos dados brutos levantados já selecionados e fragmentados. Percebeu-se que, como usuários interagem com materiais por meio dos produtos de que se utilizam em suas tarefas diárias, tal interação leva a que, com o passar do tempo, atribuam significados aos produtos com base em entendimentos decorrentes deste processo. A qualidade de um material, sob forma de produto, é, então, avaliada considerando-se fatores tais como a categoria do produto, o contexto associado, seu custo, durabilidade, resistência, acabamento e a aparência inicial versus aparência pós-uso. Este processo acontece tanto por meio de experiência individual quanto em interação com outros usuários. Por outro lado, designers operam como agentes que direcionam escolhas e configuram produtos a partir de premissas projetuais e de solicitações da indústria de bens de consumo, visando atender aos usuários. Como parte de suas atribuições, configuram produtos, selecionam materiais e indicam processos e acabamentos que thes pareçam adequados também para carregar, semanticamente, as experiências que os produtos que projetam propiciarão a seus usuários.

Palavras-chave: design de produto, usuário, semântica de produtos, semântica de materiais, materiais plásticos.

Abstract: This article presents the results of master's degree research completed in 2015. The phenomenological research, done with users and professional designers, is on the perception of the qualities of plastic 
material applied in product design. In this study, in-depth, semi-structured interviews were carried out with 35 users and 12 designers. This was an unstratified sample which included men and women of different ages. Data treatment was done using the associative data analysis technique, based on the process of inductive identification of themes, from the analytical examination of the already selected and fragmented raw data. It was observed that, because users interact with material by way of the products they use in their daily tasks, this interaction leads, over time, to attributing meanings to products based on understandings which have resulted from this process. Therefore the quality of a material, in the form of a product, is evaluated considering factors such as the product category, the associated context, its cost, durability, resistance, finishing and its initial appearance as compared to its post-use appearance. This process happens both through individual experience and through interaction with other users. On the other hand, designers operate as agents who direct choices and configure products based on project premises and on requests from the consumer goods industry, in order to cater to users. As part of their work, they configure products, select materials and indicate processes and finishings that they deem appropriate, and also to instill, semantically, the experiences that the products they design will provide to users.

Keywords: product design, user, products semantics, materials semantics, plastic materials

\section{INTRODUÇÃO}

Este estudo se ocupou de investigação do que pensam os usuários a respeito dos materiais plásticos associados aos produtos do seu dia a dia, em especial produtos pessoais e domésticos. Pesquisas a respeito da experiência dos usuários com materiais são recorrentes no campo, e dentre elas destacam-se os trabalhos de Doordan (2003); Desmet e Schifferstein (2003); Fisher (2004); Dias (2009); Karana (2009); Dias (2009); Karana e Hekkert (2010); Wastiels et all (2013); Karana, Pedgley e Rognoli (2014); Karana et al. (2015). Conforme revisão da literatura especializada do campo, "usuários somente interagem com materiais por meio de produtos" (ASHBY e JOHNSON, 2011). Através dessa interação usuário-produto-material, formulam-se entendimentos e sentidos. Plásticos são materiais que se apresentam para o design de produtos com capacidade de se transformar em tudo o que se projete. Suas capacidades de transformação são bem conhecidas. Polímeros são famílias de materiais com características muito particulares, e que, também, podem ser associadas por vezes entre elas - por exemplo, blendas poliméricas ou compostos e compósitos, objetivando aumento de desempenhos específicos.

No universo de materiais, plásticos se destacam dentre outros materiais, pela grande capacidade de transformação. Considerando-se a imensa família de materiais conhecida como polímeros, não possuem características únicas e padronizadas. Para qualquer tipo de produto, seja ele pessoal e/ou doméstico, é possível selecionar, dentre muitos, uma categoria que seja mais viável em atendimento aos requisitos de 
cada projeto. Devido a essas considerações acerca de suas propriedades, despertam percepções diferentes a cada produto e material aplicado a ele.

Tais materiais permitem um sem fim de aplicações e associações para produtos tais como: a capacidade de mimetizar outros materiais; substituir o papel desempenhado pelos materiais mais clássicos; seu baixo custo e alta escala produtiva torna-o multicontextualizado; suas percepções são híbridas, assim como a sua capacidade de transformação; há significativos reflexos sobre a nossa cultura atual, de consumismo desenfreado, contribuindo para mudança de hábitos, transformando a cultura material atual.

Outros estudos, mais recentes, examinam materiais como direcionador para o design, como em Karana et al. (2015, p. 35). Alguns estudos revisitam trabalhos anteriores, conceitos já levantados e esmiuçados por autores como Manzini (1993) segundo o qual, um material deve ser socialmente e culturalmente aceito; deve fazer sentido para os usuários; enfatizando suas qualidades funcionais; e, designers devem questionar "o que isso faz". Em uma afirmativa do autor mencionado, de que o material, suas propriedades, possibilidades de aplicações e sua performance, afetam usuários em mais de uma maneira e se reflete sobre a experiência (KARANA, 2015, p. 36) reafirma-se que a avaliação de um produto pelo usuário pode acontecer além de sua percepção utilitária.

Yanagisawa e Takatsuji (2015, p. 39) debruçaram-se sobre os efeitos da expectativa e qualidades percebidas dos materiais e texturas atribuídas à sua superfície. A esse respeito, contam que indivíduos percebem ou preveem as características de uma superfície do material correspondente a cada atributo físico, por meio de informação sensorial, que foi chamado de "atributos percebidos"; características de superfície avaliadas como qualidades tácteis daquele material. Os autores predizem que designers devem atentar para essa relação entre os atributos físicos da superfície, como parâmetros do design, e as suas respostas psicológicas, que também se relacionam à percepção da superfície, à sensação de aspereza ou suavidade durante a percepção táctil.

Silva, Crilly e Hekkert (2015) acrescentam um estudo a respeito de como as intenções de designers afetam a experiência das pessoas com produtos. Segundo eles, "produtos são resultados de processos que são conduzidos por designers. São eles que conduzem como o produto deve ser e como operam (p. 21)". Para os autores do estudo, designers projetam não apenas preocupados com intenções práticas, ou contribuem para facilitar rotinas ou a praticidade de produtos, mas para elicitar certa experiência, atitude ou comportamento de pessoas.

Materiais de um modo geral evocam atributos percebidos em forma de produtos. Não se pode obter um vocabulário que nos seja útil na formulação de linguagem e comunicação para o campo do design, sem ouvirmos aqueles a que se destina a atividade do design -os usuários, a respeito de suas percepções sobre os materiais envolvidos em um produto.

\subsection{Semântica do material}

No presente estudo, adotou-se a abordagem da teoria de significados do campo da semântica do produto, proposta por autores relatados na revisão da literatura, quais sejam: Krippendorff e Butter (2007); Karana (2009); Karana e Hekkert 
(2010). Este campo de estudo da semântica do produto, teorizado por Krippendorff e Butter (1984), é caracterizado "como o estudo das qualidades simbólicas das formas produzidas pelo homem no contexto de seu uso e a aplicação deste conhecimento para o design industrial."

Ainda a respeito do protagonismo dos plásticos, Sparke (1990, p. 11) revela que eles constituem uma família de substâncias capazes de evidenciar tanto significados de decadência como de progresso e modernidade, e, de certo modo, refletem a intersecção entre a alta cultura e a cultura de massa, traduzindo-se em um material com grande demanda e capacidade de espelhar muitos valores da sociedade atual.

Para Krippendorff (2007), os usuários, ao interagirem com materiais por meio de produtos e por intermédio de seus sentidos (visão, audição, tato, olfato e paladar), constroem significados para os objetos que os rodeiam e os materiais de que são feitos, não apenas em aspectos físicos, mas também, de acordo com o que significam para eles. Contudo, tais significados não podem ser analisados fora de seu contexto de uso e da interação com suas funções práticas.

Em outros termos, quando os usuários se servem de objetos no seu cotidiano, em atendimento às mais variadas atividades, interagem com materiais de vários modos, em diversas aplicações e variadas condições. Na maioria das vezes, podem relacionar-se com o mesmo tipo de material em circunstâncias e contextos diferentes. Estes significados podem variar entre diferentes indivíduos e diferentes culturas. Além disto, significados variam com o tempo e de acordo com a memória de experiências anteriores ou associações (GIBSON, apud HOCHBERG, 1994).

De acordo com os autores acima referidos, "sem contexto as coisas não fazem sentido para nós"; assim como para Krippendorff e Bütter, (2007, p. 4), Schiffeirstein e Hekkert (2008), Karana e Hekkert (2010), Karana (2010) e Ashby e Johnson (2011) não há regras simples para explicar a experiência usuário-produto-significados. Entender como pensam os indivíduos que se conectam com esses objetos, em seu contexto de uso e práticas, é importante para compreender como se processa a experiência; e essa compreensão é fonte de possíveis estratégias na busca por inovações e melhorias na experiência com o material de que são feitos os produtos.

Pessoas interagem diariamente com produtos; estes, por sua vez, apresentam uma mesma propriedade: a forma, que representa o limite do material por meio do qual distinguimos os objetos em nosso ambiente (MULLER apud KARANA e HEKKERT, 2010, p. 44). Os autores acima relatados definem: "A característica expressiva (ou significado) de um material é baseado na interação entre indivíduo e o produto e seu material, que pode mudar sobre o tempo".

\section{MÉTODO}

Neste estudo, foram entrevistados 35 usuários e doze designers profissionais, homens e mulheres, em amostra não estratificada e de idades variadas. As entrevistas foram realizadas em residências e locais de trabalho, na cidade de São Paulo. A técnica escolhida para a coleta de dados foi a entrevista em profundidade semiestruturada. Tal técnica, é um dos instrumentos recomendados por muitos autores para estudos dessa natureza. Por exemplo, Flick (2009, p. 143-54), que afirma que essa modalidade de entrevista é mais bem avaliada, por considerar que "é mais provável que os pontos de vista dos sujeitos entrevistados possam ser melhor expostos em planejamentos 
abertos do que em uma entrevista padronizada ou questionário".

A técnica de coleta de dados apoiou-se em um roteiro elaborado para o trabalho de campo. O roteiro se serviu de questões mais abertas, durante a entrevista, objetivando facilitar as respostas dos participantes em busca de maior espontaneidade, na exposição dos conhecimentos que possuíam a respeito da temática da pesquisa, conforme sugerido por Bauer e Gaskell (2010, p. 64-66). Os roteiros utilizados para os grupos de participantes - usuários e designers profissionais -, foram elaborados considerando-se o nível de conhecimento de cada grupo. Sendo assim, havia diferentes roteiros/tópicos de perguntas-guias, em busca de atender às indagações exploratórias da pesquisa que se pretendeu descobrir.

$\mathrm{Na}$ fase de tratamento dos dados, foram criadas categorias conceituais diferentes para os dois grupos de participantes do estudo, por causa do grau de abstração de cada um, reflexo de aspectos culturais diferentes e inerentes a cada grupo de indivíduos. Foi possível notar nítidas diferenças de entendimentos, complexidade e graus de abstração nas respostas.

Após a coleta de dados, foram realizados os procedimentos de transcrição triagem, separação e indexação dos dados analisados -, utilizando-se a técnica de análise associativa de dados, que consiste do processo de identificação indutiva de eixos temáticos, a partir do exame analítico dos dados brutos levantados, já selecionados e fragmentados. Este processo gerou categorias conceituais, empregadas para estruturar a discussão dos dados obtidos para cada grupo de participantes da pesquisa. As categorias conceituais não foram, necessariamente, as mesmas para os dois grupos de participantes, havendo categorias coincidentes e associações por aproximação. Desta maneira, foi possível identificar, em geral, significativas diferenças de percepções, complexidade e graus de abstração nas respostas entre os dois grupos de participantes.

\section{RESULTADOS}

\subsection{O conceito de qualidade de produto-material por usuários participantes}

Os dados analisados da categoria de participantes formada por usuários merecem algumas considerações sobre padrões encontrados nos dados levantados. Muitos depoimentos refletem entendimentos que se associam aos padrões encontrados na categoria formada por designers profissionais. Revelaram-se, por meio destas declarações, padrões e tendências, reflexões talvez, de repertórios culturais individuais de experiências com muitos produtos associados a amplo contexto de aplicação do plástico no design de produtos. Para este artigo analisamos os resultados destacados e sistematizados, sobretudo junto à categoria conceitual "aspectos de qualidade". Também são considerados os resultados de outras categorias conceituais que se associam ao sentido de qualidade por aproximação, sendo estas categorias relacionadas: aspectos de funcionalidade; aspectos comparativos com outros materiais; o nacional versus importado; marca como sinônimo de qualidade; aspectos de valor/custo.

Os aspectos de durabilidade parecem estar associados à avaliação táctil da resistência, rigidez ou à dureza do material aplicado ao produto, quando examinados pelos usuários ao mencionarem em seus relatos: 
Acho que no momento que você apalpa o plástico, mais ou menos você percebe a durabilidade se é bom ou ruim; [...] Sabe, quando você pega, você percebe a grossura do material, se é resistente; depois, tem uns que, sai da geladeira e vai para a mesa e vai para o micro-ondas e não deforma.

Os participantes também associam sua qualidade diretamente ligada à fabricação, à resistência do material ao longo do tempo uso e à facilidade de limpeza e manutenção. É necessário mencionar que há muitas modalidades de produtos que sequer foram mencionados pelos participantes, embora tenham sido observados no ambiente da casa durante as entrevistas.

Produtos nacionais em materiais plásticos ainda são vistos como inferiores aos competidores importados, mas este sentido não resulta em um padrão de sentido geral. Identificam-se, entre os comentários dos participantes, citações de marcas que refletem atribuições de qualidade superior a produtos importados, quando comparados aos da indústria nacional. Como no exemplo citado a seguir:

Antigamente [...] o nacional era um pouco mais rústico [...] era mais feio vai, entre aspas, assim esteticamente falando. [...] agora, tá meio que na mesma; percebo muita diferença... A beleza do produto é diferente, o acabamento é diferente e a durabilidade é diferente; Por isso eu acho que o material é diferente; acho que os importados são mais resistentes; Os brinquedos eu acho duram mais que os nacionais. Aquela marca Coza é boa, dura; tem também tupperware que eu tenho em casa que dura muito, daquela marca Tupperware mesmo, só que não vende em mercado, mas eu tenho há muito tempo.

Os nacionais, comparados aos importados, possuem na visão dos participantes um melhor desenvolvimento, percebido pela qualidade estética e do material que denota aparência melhor. Consequentemente, a percepção de durabilidade parece ser maior. Talvez seja resultado de nossa condição econômica que se reflete sobre o custo produtivo e, portanto, no preço final da peça. Neste caso, o preço final do produto é uma variável importante para a percepção da qualidade do ponto de vista do usuário.

Contudo, a percepção do usuário pode sofrer muitos estímulos no que diz respeito à qualidade: o produto em si e seu aspecto formal, seus desempenhos, bem como o material, estando associado a muitos produtos de vida curta ou de pouca durabilidade. Os plásticos, conforme dados desta pesquisa, tornaram-se um material com milhares de aplicações. A mais recorrente destas aplicações, identificada nesta pesquisa é para utilidades e utilitários de cozinha, produtos para limpeza e organização da casa. Tais produtos, em sua grande maioria, situam-se em uma faixa estimada de preço baixo ou, no máximo, preço médio; e possuem vida curta na maioria dos casos. Há grande presença destes produtos entre as categorias de produtos pessoais e domésticos.

Em consequência destes estímulos, tais produtos são vistos como de qualidade duvidosa. Para produtos destas modalidades citadas, tal percepção pode estar 
associada à facilidade com que se deteriora sua aparência estética inicial, perdendo assim seu valor e até sua utilidade. Isto acontece devido à facilidade de troca e à preços muito acessíveis, contribuindo assim para um descarte rápido, conforme fragmentos de relatos a seguir:

[...] conforme você vai lavando, você usa para pôr no freezer, muitas vezes vão rachar mais rápido. Então, não é para o modo como eu faço, lavo; Pode ser que eu esteja errada, mas o modo como eu uso, ela tem uma durabilidade menor do que a louça.

Sua beleza está associada à qualidade de sua fabricação e ao design (mesmo que não se saiba o real significado do termo). Texturas que apresentem o material plástico em determinadas modalidades de produtos ou textura, que confiram aos produtos: a sensação de aderência percebida pela avaliação táctil; brilho; polimento; e a própria espessura do material plástico, refletida em uma visão positiva do material, e que Ihe dá certa beleza, refletindo na compreensão e no significado de qualidade.

\subsection{0 conceito de qualidade de produto-material por participantes designers}

Nas análises de resultados do grupo de participantes formado por designers, identificaram-se padrões de abstração encontrados nos dados levantados. Muitos depoimentos refletem percepções que são associadas a categorias conceituais do grupo de participantes usuários (aspectos de qualidade/durabilidade; preço versus custo). Revelam-se padrões e tendências de entendimentos, reflexos de suas práticas profissionais. O conceito de qualidade para designers está associado ao material associado aos produtos, e, depende da segmentação de mercado e custo final que o fabricante/indústria quer alcançar. As categorias associadas à qualidade para os designers são: perda de valor versus aspectos de qualidade; custo versus qualidade; cópia versus qualidade.

Depoimentos dos participantes designers a respeito do que pensam os usuários sobre os materiais plásticos, aplicados ao design de produtos, em linhas gerais, refletem percepções que são resultado de suas ações projetuais. Isto significa que designers pensam sobre plásticos em aspectos associados ao projeto como um todo. A preocupação com o que pensam os usuários é uma informação que lhes chega na qualidade de público-alvo.

[...] a questão de escolha do material; primeiro a gente tem que focar na área que vai atuar; pensando no consumidor, pensamos no que ele quer comprar; tem ainda questões de custo, se, o cliente quer um produto barato; se o material vai dar um bom acabamento; se vai dar uma boa textura.

Designers reconhecem estratégias de projeto e processos de produção afim de baixar os custos de um produto. Nota-se, reiteradas vezes, a menção de redução da espessura de paredes; a diminuição do tempo do ciclo da injeção plástica; a substituição de um polímero específico de custo mais alto, de melhor qualidade, por um de custo mais baixo - constituindo assim um rebaixamento da qualidade de 
material e do produto, a fim de reduzir custos. Mencionou-se também a produção realizada na China, a preços muito baixos, com o objetivo de reduzir o custo e o preço final. O custo final solicitado de produção é fator que define a qualidade da matériaprima utilizada e do produto.

Quando o consumidor está disposto a pagar barato por ele (produto), é o plástico que permite isso; senão, não poderia ter esse ou aquele produto; ou talvez não pudesse comprar; isso é muito claro para mim; não adianta falar que produto da China é porcaria.

Designers profissionais demonstraram certa dificuldade em identificar-se na posição de usuários. Quando solicitados que comentassem "o que eles achavam que usuários pensam a respeito dos materiais plásticos aplicados a produtos", seus depoimentos revelaram preocupações e requisitos de natureza projetual. Muitos relatos apontam nesta direção. Talvez estas preocupações resultem das suas práticas profissionais e reflitam-se em suas respostas. Ou poderia ser também a ausência de conhecimentos adquiridos por meio de pesquisas qualitativas, realizadas junto a usuários e a respeito de suas concepções sobre produtos e materiais.

O custo faz parte do negócio; mas a gente não pode nunca abrir mão da qualidade; tenho um limite para pagar, um limite de investimento no molde; depois limite de produção, no tipo de plástico que eu vou usar. É o consumidor que está disposto a pagar aquele preço, por aquele produto; os projetos já nos chegam, em geral, com o material definido.

Como resultados da análise dos dados, percebe-se que, quanto à durabilidade do material, designers acreditam que o material plástico possui todas as propriedades necessárias para atender aos requisitos de projeto. Porém, reiteram que entregam o projeto para o cliente com todas as especificações de projeto e material a ser aplicado, mas que às vezes pode acontecer de o cliente fazer alterações de material com o propósito de baixar seus custos de produção. Desta forma, a qualidade decai diante do fator do preço final a ser alcançado.

Em consequência de esforços para baixar custos e ampliar a competitividade dos produtos em um mercado economicamente instável, materiais de maior qualidade são substituídos, dando lugar a outros inferiores; espessuras são diminuídas e talvez, não se invista tanto no desenvolvimento de produtos, utilizando-se das expertises de designers. Pode ser mais interessante sobrepor etapas e copiar produtos a um custo menor.

O grau de mortalidade de produtos é alto; algo em torno de $85 \%$ para produtos novos. Quando se copia um produto, primeiro, se elimina o sistema de pesquisa, segundo, o problema de aceitação [...] o risco é menor, já que na indústria é muito alto economicamente falando. 


\section{CONSIDERAÇÕES FINAIS}

Há muitas visões dos usuários a respeito de suas percepções sobre produtos plásticos, que são reflexos do direcionamento de designers e das indústrias, estando associadas aos requisitos projetuais e seleção de materiais. Alguns aspectos mais relevantes dizem respeito à perda de durabilidade/qualidade dos produtos, decorrentes do rebaixamento de valores de materiais e produtos, com objetivo de atingir competitividade de mercado. Isto ocorre segundo uma visão do que é desejável (qualidade e preço), porém viável (baixo custo produtivo e preço acessível), com o objetivo da permanência de produtos e, consequentemente, da indústria, no mercado cada vez competitivo e de momento econômico instável.

Embora o designer sempre tenha a prerrogativa de se posicionar a respeito dos materiais mais adequados aos produtos que desenvolve, pensando na qualidade final destes, a decisão final costuma caber ao cliente que o contrata. O cliente, ou seja, a indústria responsável pela produção, deveria perfilhar a visão do designer, que pensa efetivamente na qualidade do produto final, considerando o usuário final e o que ele quer comprar. No entanto, as decisões são refletidas no resultado final do produto. Isto poderá traduzir-se em um produto que denote baixa qualidade.

Objetivando lucros por meio de grande volume de vendas, são selecionados materiais de custo menor, bem como espessuras reduzidas, ciclos de processamento também reduzidos e critérios de seleção questionáveis, do ponto de vista da confiabilidade, de modo a não conter contaminantes em sua composição. Neste contexto, a cópia se torna prática aceitável e justificada pelo risco menor de investimento (o que não afeta diretamente os designers). Não se mencionam pesquisas realizadas diretamente com usuários. Não são reveladas preocupações com a experiência do usuário com o produto, sendo que, em uma visão estratégica, ouvir e analisar o que os usuários pensam poderia servir como subsídio para a melhoria de produtos, tanto em aspectos formais, de funcionalidade e praticidade, quanto em aspectos estéticos, de durabilidade e de experiência.

Os resultados apontam para uma acentuada estratificação em classes socioeconômicas de significados atribuídos a produtos em plástico. Diferentes categorias de produtos, faixas de preços, segmentação de mercado, públicos diferenciados, todos são fatores ou variáveis a serem consideradas que parecem afetar os entendimentos e atribuições de significados a produtos e materiais. Reiteradas vezes, nos resultados com usuários participantes, foi mencionado que a qualidade do produto está associada a sua manufatura e ao design, além de uma visão geral dos aspectos formais. Ficou evidente a comparação com produtos de mesma categoria, porém em outros materiais que não o plástico. Sua qualidade é comparada com a durabilidade, a praticidade, a boa higienização e manutenção atribuídas a outros materiais.

Polímeros são parte integrante de nossa cultura material atual, que atualmente não sobrevive sem eles. São materiais de relevância para designers, e de elevada complexidade projetual no tocante à suas aplicações. Muitas são as diretrizes que orientam a fabricação de produtos com estes materiais, não somente em aspectos projetuais e técnicos. É desejável que se associem, de maneira integrada, diretrizes projetuais que considerem o que realmente pensam os usuários. Que sejam trazidos, para as discussões iniciais de projetos, resultados que dêem subsídios para que os 
designers projetem: a experiência e as possibilidades de melhoria, ouvindo diretamente aqueles para os quais o resultado de suas atividades são direcionados os usuários. Pesquisas que abordem a dimensão semântica de objetos de design, segundo a perspectiva dos usuários, oferecem importante subsídio a este processo.

\section{REFERÊNCIAS}

ASHBY, Michael e JOHNSON, Kara. Materiais e design: Arte e ciência da seleção de materiais no design de produto. Rio de Janeiro: Elsevier, 2011.

BAUER, Martin. W. e GASKELL, George. Pesquisa qualitativa com texto, imagem e som: um guia prático. Rio de Janeiro: Vozes, 2010.

DIAS, Maria Regina Alves Correia. Percepções dos materiais pelos usuários: modelo de avaliação Permatus. Tese de Doutorado. Engenharia e Gestão do Conhecimento da Universidade Federal de Santa Catarina: Florianópolis, 2009.

DESMET, Pete M.A e SCHIFFERSTEIN, H.N.J. Tools facilitating multi-sensory product design for all aspects. The Design Journal: A International Journal for All Aspects of Design, Vol.11, Issue 2, 2008.

DESMET, Pete M.A e HEKKERT, Paul. Framework of product experience. International Journal of Design, vol. 1, n.1, 2007.

DESMET, Pete M.A. A multilayered model of product emotions. The Design Journal: A International Journal for All Aspects of Design, Vol.6, Issue 3, 2003.

DOORDAN, P.D. On materials. In: Design Issues, 19 (Autumm, 2003): p. 3-8, MIT Press. FAUCHEAU, Jenny; DEL CURTO, Bárbara; DELAFOSSE, David. Experimental setup for visual and tactile evaluation of materials and products through Napping. Disponivel em: <https://www.researchgate.net/ publication/280935322>, 2015, p. 1-2.

FISHER, Tom.H. What we touch, touch us: Materials, affects, and affordances. In: Design Issues, 20 (Autumm, 2004): p. 20-31. MIT Press.

FLICK, Uwe. Introdução à pesquisa qualitativa. Porto Alegre: Artmed, 2009.

KARANA, Elvin. Meaning of material. Master of Industrial Design. Midle East Tchnical University,Turkey, 2009.

How do materials obtain their meanings? Middle East Technical University (Ankara) Turkey, 2010. Disponível em: <http://jfa.arch.metu.edu.tr/archive/02585316/2010/ciet27/sayi_2/271-285.pdf>.

KARANA, Elvin; HEKKERT, Paul. User-material-product Interrelationships in Attributing Meanings. International Journal of Design, Vol.4, no. 3, 2010. Disponível em: $<$ www.ijdesign.org/>, acesso em 7/2/2013.

KARANA, Elvin; PEDGLEY, Owen; ROGNOLI, Valentina. Materials Experience: Fundamentals of materials and design. Oxford, 2014.

HOCHBERG, J. James Jerome Gibson: A biographical memoir by Julian Hochberg. National Academy of Sciences Washignton D.C., 1994.

KATZ, Sylvia. Plastics: Commom objects, classic design. Londres: Thames and Hudson, 1984. 
KRIPPENDORFF, Karl. e BUTTER, Reinhard. Product Semantics: Exploring the symbolic qualities of form. Annenberg School for Communication: University of Pennsylvania, 1984. Disponível em: <http:repository.upenn.edu/asc_papers/40>, acesso em 3/07/2013.

Semantics: Meanings and contexts of artifacts. Annenberg School for Communication: University of Pennsylvania, 2007.

On the essential contexts of artifacts or on the proposition that “Design is make sense (of things)". Design Issues: Vol. V, no. 2, Spring 1989.

Propositions of Human-centeredness: A Philosophy for design. Annenberg School for communication: University of Pennsylvania, 2000. Disponível em: <http:repository.upenn.edu/asc_papers/210>, acesso em 3/07/2013.

MOSSMAN, Susan. Fantastic plastic: Product design + consumer culture. Londres: Black Dog, 2008.

ROSS, P. e WENSVEEN, S.A.G. Design behavior in interaction: Using aesthetic experience as a Mechanism for design. International Journal of Design, Vol.4, no. 2, 2010. Disponível em: <www.ijdesign.org>, acesso em 1/mar/2014.

SPARKE, Penne. The plastic age: From modernity to post-modernity. Londres: Victoria e Albert Museum, 1990.

SILVA, O.; CRILLY, N.; HEKKERT, Paul. How people's appreciation of products is affected by their knowledge of designers' intentions. In: International Journal of Design, Vol. 9 No. 2, 2015.

YANAGISAWA, H e HAKATSUJI, K. Effects of visual expectation on perceived tactile perception: An evaluation method of surface texture with expectation effect. Disponível em: <www.ijdesign.org>, International Journal of Design Vol. 9 No. 1. acessado em 15/ jan/ 2015. 